\title{
A Short Note on the Robust Combinatorial Optimization Problems with Cardinality Constrained Uncertainty
}

\author{
Taehan Lee \\ Department of Industrial and Information Systems Engineering \\ Chonbuk National University, Korea \\ Changhyun Kwon* \\ Department of Industrial and Systems Engineering \\ University at Buffalo, SUNY, USA
}

July 23, 2014

\begin{abstract}
Robust combinatorial optimization problems with cardinality constrained uncertainty may be solved by a finite number of nominal problems. In this paper, we show that the number of nominal problems to be solved can be reduced significantly.
\end{abstract}

Keywords: robust combinatorial optimization; discrete optimization

\section{Robust combinatorial optimization problem}

Let $X \subset\{0,1\}^{n}$ be a set of feasible solutions of a combinatorial optimization problem. The nominal combinatorial optimization problem of our interest is defined as follows:

$$
\min _{\boldsymbol{x} \in X} \boldsymbol{c}^{\top} \boldsymbol{x}
$$

where $\boldsymbol{c}^{\top} \boldsymbol{x}=\sum_{j=1}^{n} c_{j} x_{j}$. Bertsimas and Sim (2003) considered uncertainty for objective coefficients such that the cost of item $j \in N=\{1,2, \cdots, n\}$ takes a value in the interval $\left[c_{j}, c_{j}+d_{j}\right]$, where $d_{j} \geq 0$. A robust combinatorial optimization problem is considered in the following form:

$$
Z^{*}=\min _{\boldsymbol{x} \in X}\left\{\boldsymbol{c}^{\top} \boldsymbol{x}+\max _{\{S|S \subset N,| S \mid \leq \Gamma\}} \sum_{j \in S} d_{j} x_{j}\right\}
$$

where at most $\Gamma$ components of the cost coefficients can be $c_{j}+d_{j}$; hence, the uncertainty set is called cardinality constrained. The budget of uncertainty $\Gamma$ is a positive integer and represents the risk attitude of decision makers, and $1 \leq \Gamma \leq n$. Without loss of generality, we assume that the indices are sorted in descending order of the size of $d_{i}$ and define $d_{n+1}=0$ so that

$$
d_{1} \geq d_{2} \geq \cdots \geq d_{n} \geq d_{n+1}=0 .
$$

Bertsimas and Sim (2003) showed that 22 is equivalent to

$$
Z^{*}=\min _{\boldsymbol{x} \in X, \theta \geq 0}\left(\Gamma \theta+\boldsymbol{c}^{\top} \boldsymbol{x}+\sum_{j \in N} \max \left(d_{j}-\theta, 0\right) x_{j}\right)
$$

\footnotetext{
*Corresponding Author: chkwon@buffalo.edu
} 
and it can be solved by solving $n+1$ nominal problems. In particular,

$$
Z^{*}=\min _{l=1,2, \cdots, n+1} G^{l}
$$

where for $l=1,2, \ldots, n+1$ :

$$
G^{l}=\Gamma d_{l}+\min _{\boldsymbol{x} \in X}\left(\boldsymbol{c}^{\top} \boldsymbol{x}+\sum_{j=1}^{l}\left(d_{j}-d_{l}\right) x_{j}\right)
$$

We let $x_{n+1}=0$, so that $(6)$ is well-defined.

This result is very useful, because we can solve the robust optimization problem by solving a finite number of nominal problems. If the nominal problem can be solved in polynomial time, we can also solve the corresponding robust problem in polynomial time. Park and Lee (2007) showed that the number of nominal problems to be solved can be reduced to $n-\Gamma+1$, and Álvarez-Miranda et al. (2013) to $n-\Gamma+2$ independently. In this paper, we show that the number of nominal problems to be solved can be further reduced to $\left\lceil\frac{n-\Gamma}{2}\right\rceil+1$.

\section{New Results}

For a feasible solution $\boldsymbol{x} \in X$, let

$$
G^{l}(\boldsymbol{x})=\Gamma d_{l}+\boldsymbol{c}^{\top} \boldsymbol{x}+\sum_{j=1}^{l}\left(d_{j}-d_{l}\right) x_{j} \quad \forall l=1,2, \ldots, n+1
$$

then $G^{l}$ in $(6)$ can be written as

$$
G^{l}=\min _{\boldsymbol{x} \in X} G^{l}(\boldsymbol{x})
$$

We let $G^{0}(\boldsymbol{x})=G^{1}(\boldsymbol{x})$ for notational simplicity. We also define $\boldsymbol{x}^{l} \in X$ such that

$$
G^{l}=G^{l}\left(\boldsymbol{x}^{l}\right) \leq G^{l}(\boldsymbol{x}) \quad \forall \boldsymbol{x} \in X
$$

We first consider $G^{l+1}(\boldsymbol{x})-G^{l}(\boldsymbol{x})$ for $l=1, \ldots, n$ and $\boldsymbol{x} \in X$ :

$$
\begin{aligned}
G^{l+1}(\boldsymbol{x})-G^{l}(\boldsymbol{x})= & \Gamma d_{l+1}+\sum_{j=1}^{n} c_{j} x_{j}+\sum_{j=1}^{l+1}\left(d_{j}-d_{l+1}\right) x_{j} \\
& \quad-\Gamma d_{l}-\sum_{j=1}^{n} c_{j} x_{j}-\sum_{j=1}^{l}\left(d_{j}-d_{l}\right) x_{j} \\
= & \Gamma\left(d_{l+1}-d_{l}\right)+\sum_{j=1}^{l}\left(d_{l}-d_{l+1}\right) x_{j} \\
= & \left(d_{l+1}-d_{l}\right)\left(\Gamma-\sum_{j=1}^{l} x_{j}\right)
\end{aligned}
$$

Similarly, we consider for $l=2, \ldots, n+1$ and $\boldsymbol{x} \in X$ :

$$
G^{l}(\boldsymbol{x})-G^{l-1}(\boldsymbol{x})=\left(d_{l}-d_{l-1}\right)\left(\Gamma-\sum_{j=1}^{l-1} x_{j}\right)
$$

Using (10) and 11, we provide the following lemmas.

Lemma 1. For $l=1,2, \ldots, n$ and for any $\boldsymbol{x} \in X$, the following holds:

1. If $\sum_{j=1}^{l} x_{j} \leq \Gamma$, then $G^{l-1}(\boldsymbol{x}) \geq G^{l}(\boldsymbol{x}) \geq G^{l+1}(\boldsymbol{x})$. 
2. If $\sum_{j=1}^{l} x_{j}>\Gamma$, then $G^{l-1}(\boldsymbol{x}) \leq G^{l}(\boldsymbol{x}) \leq G^{l+1}(\boldsymbol{x})$.

Proof. Recall that $\boldsymbol{x}$ is binary.

1. We prove the first part.

(a) If $\sum_{j=1}^{l} x_{j} \leq \Gamma$, we obtain $G^{l+1}(\boldsymbol{x}) \leq G^{l}(\boldsymbol{x})$ from 10 , since $d_{l+1} \leq d_{l}$.

(b) If $\sum_{j=1}^{l} x_{j} \leq \Gamma$, then $\sum_{j=1}^{l-1} x_{j} \leq \Gamma$. Therefore we obtain $G^{l}(\boldsymbol{x}) \leq G^{l-1}(\boldsymbol{x})$ from (11), since $d_{l} \leq d_{l-1}$.

Hence, the first part is proved.

2. We can similarly prove the second part.

(a) If $\sum_{j=1}^{l} x_{j}>\Gamma$, we obtain $G^{l+1}(\boldsymbol{x}) \geq G^{l}(\boldsymbol{x})$ from 10 , since $d_{l+1} \leq d_{l}$.

(b) If $\sum_{j=1}^{l} x_{j}>\Gamma$, then $\sum_{j=1}^{l} x_{j} \geq \Gamma+1$, and consequently, $\sum_{j=1}^{l-1} x_{j} \geq \Gamma$. Therefore we obtain $G^{l}(\boldsymbol{x}) \geq G^{l-1}(\boldsymbol{x})$ from (11), since $d_{l} \leq d_{l-1}$.

This completes the proof.

Lemma 2. For any $\boldsymbol{x} \in X$, we have $G^{1}(\boldsymbol{x}) \geq G^{2}(\boldsymbol{x}) \geq \cdots \geq G^{\Gamma}(\boldsymbol{x}) \geq G^{\Gamma+1}(\boldsymbol{x})$. Furthermore, $G^{1} \geq G^{2} \geq$ $\ldots \geq G^{\Gamma} \geq G^{\Gamma+1}$.

Proof. 1. For any $l \leq \Gamma$, we have $\sum_{j=1}^{l} x_{j} \leq \Gamma$, and therefore $G^{l}(\boldsymbol{x}) \geq G^{l+1}(\boldsymbol{x})$ by the first part of Lemma 1. This completes the proof for the first part.

2. By definition (9), we have

$$
G^{\Gamma+1}=G^{\Gamma+1}\left(\boldsymbol{x}^{\Gamma+1}\right) \leq G^{\Gamma+1}(\boldsymbol{x}) \quad \forall \boldsymbol{x} \in X
$$

Choosing $\boldsymbol{x}=\boldsymbol{x}^{\Gamma}$ and applying the first part of this lemma, we obtain

$$
G^{\Gamma+1}\left(\boldsymbol{x}^{\Gamma+1}\right) \leq G^{\Gamma+1}\left(\boldsymbol{x}^{\Gamma}\right) \leq G^{\Gamma}\left(\boldsymbol{x}^{\Gamma}\right)
$$

consequently, $G^{\Gamma+1} \leq G^{\Gamma}$. By repeating the same procedure for $\Gamma-1, \Gamma-2, \ldots, 1$, we obtain the lemma.

Lemma 2 indicates that the $l=1,2, \ldots, \Gamma$ cases are no better than the $l=\Gamma+1$ case. Therefore the $l=1,2, \ldots, \Gamma$ cases need not be examined in (5), if the $l=\Gamma+1$ case is ensured to be examined.

Lemma 3. For any $l=1,2, \ldots, n$, we have either $G^{l} \geq G^{l+1}$ or $G^{l} \geq G^{l-1}$.

Proof. 1. Suppose $\sum_{j=1}^{l} x_{j}^{l} \leq \Gamma$. By definition (9)

$$
G^{l+1}=G^{l+1}\left(\boldsymbol{x}^{l+1}\right) \leq G^{l+1}(\boldsymbol{x}) \quad \forall \boldsymbol{x} \in X
$$

Choose $\boldsymbol{x}=\boldsymbol{x}^{l}$ and apply the first part of Lemma 1. Then,

$$
G^{l+1}\left(\boldsymbol{x}^{l+1}\right) \leq G^{l+1}\left(\boldsymbol{x}^{l}\right) \leq G^{l}\left(\boldsymbol{x}^{l}\right)
$$

Therefore, $G^{l+1} \leq G^{l}$.

2. If $\sum_{j=1}^{l} x_{j}^{l}>\Gamma$, we can similarly show that $G^{l-1} \leq G^{l}$, by considering $G^{l-1}$ and applying the second part of Lemma 1.

Since the two cases are mutually exclusive, we obtain the lemma.

Lemma 3 provides a way to significantly reduce the number of nominal problems to be solved; it indicates that any $l$ is no better than either $l-1$ or $l+1$. This also indicates that the minimum of $G^{l}$ occurs at two or more consecutive indices $l$, unless it does at $l=\Gamma+1$ or $l=n+1$. Our main result follows. 
Table 1: Comparison of $\mathcal{L}$, with an example of $n=20$ and $\Gamma=5$

\begin{tabular}{|c|c|c|}
\hline Authors & $\overline{\mathcal{L}}$ & $|\mathcal{L}|$ \\
\hline Bertsimas and Sim (2003) & $\begin{array}{l}\{1,2, \ldots, n+1\} \\
\quad=\{1,2,3, \ldots, 19,20,21\}\end{array}$ & $n+1=21$ \\
\hline Álvarez-Miranda et al. (2013) & $\begin{array}{r}\{\Gamma, \Gamma+1, \ldots, n-1, n, n+1\} \\
\quad=\{5,6,7, \ldots .19,20,21\}\end{array}$ & $n+2-\Gamma=17$ \\
\hline Park and Lee (2007) & $\begin{array}{c}\{\Gamma, \Gamma+1, \ldots, n-1, n+1\} \\
\quad=\{5,6,7, \ldots ., 19,21\}\end{array}$ & $n+1-\Gamma=16$ \\
\hline Theorem 1 & $\begin{array}{r}\{\Gamma+1, \Gamma+3, \Gamma+5, \ldots, \Gamma+\gamma, n+1\} \\
\quad=\{6,8,10,12,14,16,18,20,21\}\end{array}$ & $\left.\frac{n-\Gamma}{2}\right]+1=9$ \\
\hline
\end{tabular}

Theorem 1. The robust combinatorial optimization problem (2) can be solved by $\left\lceil\frac{n-\Gamma}{2}\right\rceil+1$ number of nominal problems. In particular,

$$
Z^{*}=\min _{l \in \mathcal{L}} G^{l}
$$

where $\mathcal{L}=\{\Gamma+1, \Gamma+3, \Gamma+5, \ldots, \Gamma+\gamma, n+1\}$ and $\gamma$ is the largest odd integer such that $\Gamma+\gamma<n+1$.

Proof. The set of indices $\mathcal{L}$ is obtained by Lemmas 2 and 3 . We prove the number of nominal problems to be solved. If we let $\gamma=2 k-1$, then $k$ is the largest integer such that $\Gamma+(2 k-1)<n+1$, or $k<\frac{n-\Gamma}{2}+1$; therefore $k=\left\lceil\frac{n-\Gamma}{2}\right\rceil$. Consequently, the cardinality of the set $\mathcal{L}$ is $k+1=\left\lceil\frac{n-\Gamma}{2}\right\rceil+1$. This completes the proof.

Note that in Theorem 11. the set $\mathcal{L}$ includes the two boundary indices $l=\Gamma+1$ and $l=n+1$. We compared our result with the previous results in Table 1 with an example of $n=20$ and $\Gamma=5$.

\section{Concluding Remarks}

In this short note, we showed that the number of nominal problems to be solved can be significantly reduced to obtain a solution of robust combinatorial problems. We would like to close this note by providing a small tip for further reduction that is suggested in Kwon et al. (2013) for the case when the cost vector $\boldsymbol{c}$ is nonnegative. Suppose $G^{\sharp}$ is the smallest $G^{l}$ found so far. Then, there is no need to consider any indices $l$ such that $\Gamma d_{l} \geq G^{\sharp}$, since the objective function value of the corresponding nominal problem is nonnegative, hence there is no chance of improving. Therefore, by examining the set $\mathcal{L}$ in descending order, i.e., first considering $n+1$ and then $\Gamma+\gamma$ to $\Gamma+1$, we can stop when we encounter the case of $\Gamma d_{l} \geq G^{\sharp}$ for the first time.

\section{References}

Álvarez-Miranda, E., Ljubić, I., and Toth, P. (2013). A note on the bertsimas \& sim algorithm for robust combinatorial optimization problems. 4OR, 11(4):349-360.

Bertsimas, D. and Sim, M. (2003). Robust discrete optimization and network flows. Mathematical Programming, 98(1):49-71.

Kwon, C., Lee, T., and Berglund, P. (2013). Robust shortest path problems with two uncertain multiplicative cost coefficients. Naval Research Logistics, 60(5):375-394.

Park, K. and Lee, K. (2007). A Note on Robust Combinatorial Optimization Problem. International Journal of Management Science, 13(1):115-119. 